\title{
FDTD Analysis for Light Passing Through Glass Substrate and Its Application to Organic Photovoltaics with Moth Eye Antireflection Coating
}

\author{
Shigeru Kubota ${ }^{1, *}$, Kensaku Kanomata ${ }^{1}$, Bashir Ahmmad ${ }^{1}$, Jun Mizuno ${ }^{2}$, \\ and Fumihiko Hirose ${ }^{1}$ \\ ${ }^{1}$ Graduate School of Science and Engineering, Yamagata University, \\ 4-3-16 Jonan, Yonezawa, Yamagata 992-8510, Japan \\ ${ }^{2}$ Research Organization for Nano and Life Innovation, Waseda University, \\ 513 Wasedatsurumaki-cho, Shinjyuku-ku, Tokyo 162-0041, Japan \\ *kubota@yz.yamagata-u.ac.jp
}

\begin{abstract}
To improve the performance of organic photovoltaics (OPVs), it is useful to trap light by using the antireflection nanotexture such as moth eye structure. The finite-difference time-domain (FDTD) method is frequently used to analyze the optical properties of nanotexture. However, in the case that FDTD is applied to OPVs, the existence of a glass substrate generates a strong oscillation in optical response, which does not exist in the actual device. To remove such oscillatory components and accurately simulate optical response, we study an FDTD-based computational algorithm, which we call the envelope method. We compare this method with other possible methods, and demonstrate that the envelope algorithm is more accurate for estimating optical response and more robust against parameter variations than the other ones. We also apply this method to analyze the changes in the OPV performance associated with the changes in the properties of moth eye coating.
\end{abstract}

Keywords: organic solar cells, antireflection, optical simulation, FDTD

\section{Introduction}

Organic photovoltaics (OPVs) have been attracting high attention, because of their potential for cost-effective, large-area, and flexible solar cells in the next generation [1, 2]. The performance of OPVs has been significantly increasing in the last decade [3], although further increase in the power conversion efficacy will be necessary for large-scale application. Since the OPV device contains a stack of several thin layers, a certain portion of light energy is always lost by the reflection at the interfaces between adjacent layers and the absorption in the layers different from the active layer [4]. To decrease these optical losses, it is needed to adequately design antireflection (AR) coating that serves to trap light into the device.
Recently, many studies on the AR structure (ARS) have investigated moth eye texture, which is a two-dimensional regular array of cones having a period and height of several hundred nanometers [4-7]. The reflection from the moth eye surface is reduced because the effective refractive index continuously changes along the axis of cones. The fabrication of large-area moth eye texture can be achieved cost-effectively by the nanopatterning techniques such as nanoimprint lithography [8].

The AR effect of moth eye coating can be analyzed by the optical simulations using the finite-difference time-domain (FDTD) method [9]. In FDTD, both space and time are divided into discrete points, and the spatiotemporal distribution of electromagnetic field is 
calculated through numerical integration. Since the FDTD method has a high modeling capability and can readily treat arbitrary nanotexture pattern, this method has a wide range of applications [7,9,10]. However, when the FDTD method is applied to OPVs, the large thickness of glass substrate, in the front side of the device, causes strong oscillatory response which does not occur in actual devices [11].

Therefore, in our previous work, we proposed an FDTD-based computational algorithm by which the optical response of the light passing through a glass substrate is efficiently obtained from FDTD simulations [12]. We used this method for analyzing the AR performance of moth eye coating for OPVs and, additionally, for finding the optimized geometric parameters of moth eye texture.

The present study extends our previous study with new results. We closely analyze our previously proposed algorithm, and compare it with the other possible algorithms. We show that our method is not only more precise in estimating the optical response of OPVs but also more robust against parameter variations, as compared to the other methods. The relevance of the algorithms is checked by comparing the obtained results with the results expected from the characteristic matrix-based analysis [13]. The results in this study suggest that the proposed method is helpful to extend our ability to analyze and design the ARS for OPVs.

\section{Experimental}

\subsection{Model structure}

We performed the optical analysis of OPVs by using the model in Fig. 1. The OPV cell is deposited on a glass substrate, and the light passes through the substrate to enter the cell. The device contains a 100 -nm-thick film of poly(3-hexylthiophene) (P3HT) and $[6,6]$-phenyl- $\mathrm{C}_{61}$-butyric acid methyl ester (PCBM) blend and a 7-nm $\mathrm{MoO}_{3}$ film, which are used as the active and hole transporting layers, respectively. These films are sandwiched between the two electrodes formed by a 100-nm indium-doped tin oxide (ITO) layer and a 100-nm Al layer. In the moth eye coating, the cones are hexagonally arranged on the front surface of the device.
For the FDTD simulations, the optical model is assumed to have a $5-\mu \mathrm{m}$-thick glass substrate, which is sufficient to make accurate prediction for the optical response (see Results and Discussion).

\subsection{Optical simulation}

We performed optical simulations using the FDTD method and characteristic matrix-based method, both of which are described in detail in our previous study [12]. Briefly, in the FDTD simulations, the absorbing boundary conditions (ABCs) are introduced, by using the perfectly matched layer approach [9]. The usage of $\mathrm{ABC}$ is needed to eliminate the reflection occurring at the boundary of the FDTD domain. In order to calculate broadband response from single simulation run, the result generated by a short pulse of incident light is Fourier transformed. The optical data for the materials used are fitted by the Lorentz-Drude model [14], which enables efficient FDTD simulations for dispersive materials.

In the characteristic matrix-based simulations [13], the optical properties of moth eye array are described with the effective medium theory (EMT) [15]. The EMT is widely applied to describe the optical properties of nanotexture in cases where the period of texture is smaller than the wavelength of the incident light. By applying EMT, the model in Fig. 1 can be considered to consist of a thick glass substrate and two thin-film multilayer stacks, which correspond to the moth eye and OPV cell. In each stack, the light waves are added coherently

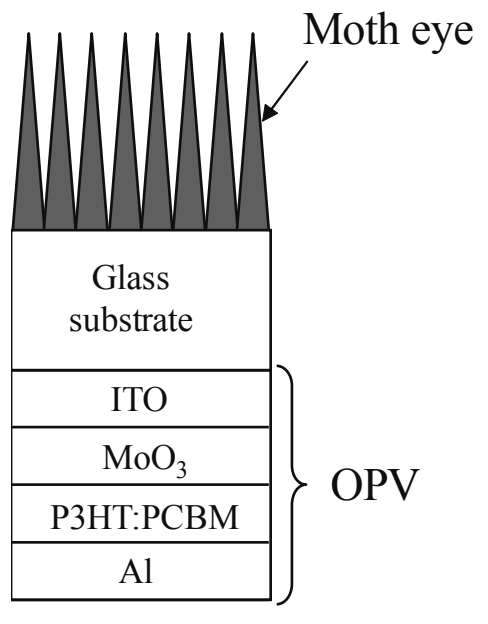

Fig. 1. Optical model of the OPV cell with moth eye coating. 
according to the characteristic matrix algorithm [13], whereas in the thick glass substrate, the light irradiances are added due to the loss of coherence.

In both the FDTD and characteristic matrix-based analysis, the short-circuit current density $\left(J_{S C}\right)$ of OPVs can be obtained as follow [12]. If we define $A_{p}(\lambda)$ to be the absorbance in the active layer at wavelength $\lambda$, the number of absorbed photons, $N_{p}(\lambda)$, is represented as

$$
N_{p}(\lambda)=A_{p}(\lambda) F(\lambda) \frac{\lambda}{h c},
$$

where $F(\lambda)$ is the AM1.5 solar irradiance spectrum, $h$ is Planck's constant, and $\mathrm{c}$ is the light speed in free space. The level of photocurrent can be calculated by the following equation:

$$
J_{S C}=\int_{0}^{\lambda_{g}} q_{e} N_{p}(\lambda) F_{N R}(\lambda) d \lambda .
$$

Here, $\lambda_{g}$ is the wavelength corresponding to the band-gap energy of P3HT $(653 \mathrm{~nm}), q_{e}$ is the unit charge. $F_{N R}(\lambda)$ is a function representing the nonrecombination factor, which is simply assumed to be $F_{N R}(\lambda)=1$ for all $\lambda$ [16].

\subsection{The algorithm for removing artificial} interference from FDTD response

In the FDTD simulations with a thick glass substrate, strong oscillatory response takes place, due to the interference of light reflecting at the front and back sides of the substrate [11,12]. An example of such oscillation, in the absorbance in the active layer, is shown in Fig. 2A (black solid line). This type of oscillation is 'artificial' in the sense that it does not exist in the actual device. This is because, in the substrate much thicker than the wavelength of incident light, the coherence is lost so that the addition of irradiances takes place, as mentioned above.

To remove such artificial interference effect and obtain correct optical response, our previous study has proposed a computational algorithm, which we call the envelope method from now on [12]. Let us define $r(\lambda)$ to be the oscillatory FDTD response, which is a function of wavelength $\lambda$ (Fig. 2A, black solid line). The local peaks and troughs of $r(\lambda)$ represent the states where the value of $r$ is maximally strengthened and weakened, respectively, by the interference of light passing through the substrate. Therefore, we can expect that the averaging between the states corresponding to the peaks and troughs results in the cancellation of the effect of artificial interference. To do this, we plot the upper and lower envelopes of $r(\lambda)$ (Fig. 2A, dashed lines) by using the spline interpolation of the peaks and troughs, and take an average between the two envelopes. The detail of the envelope method is described in the following three steps:
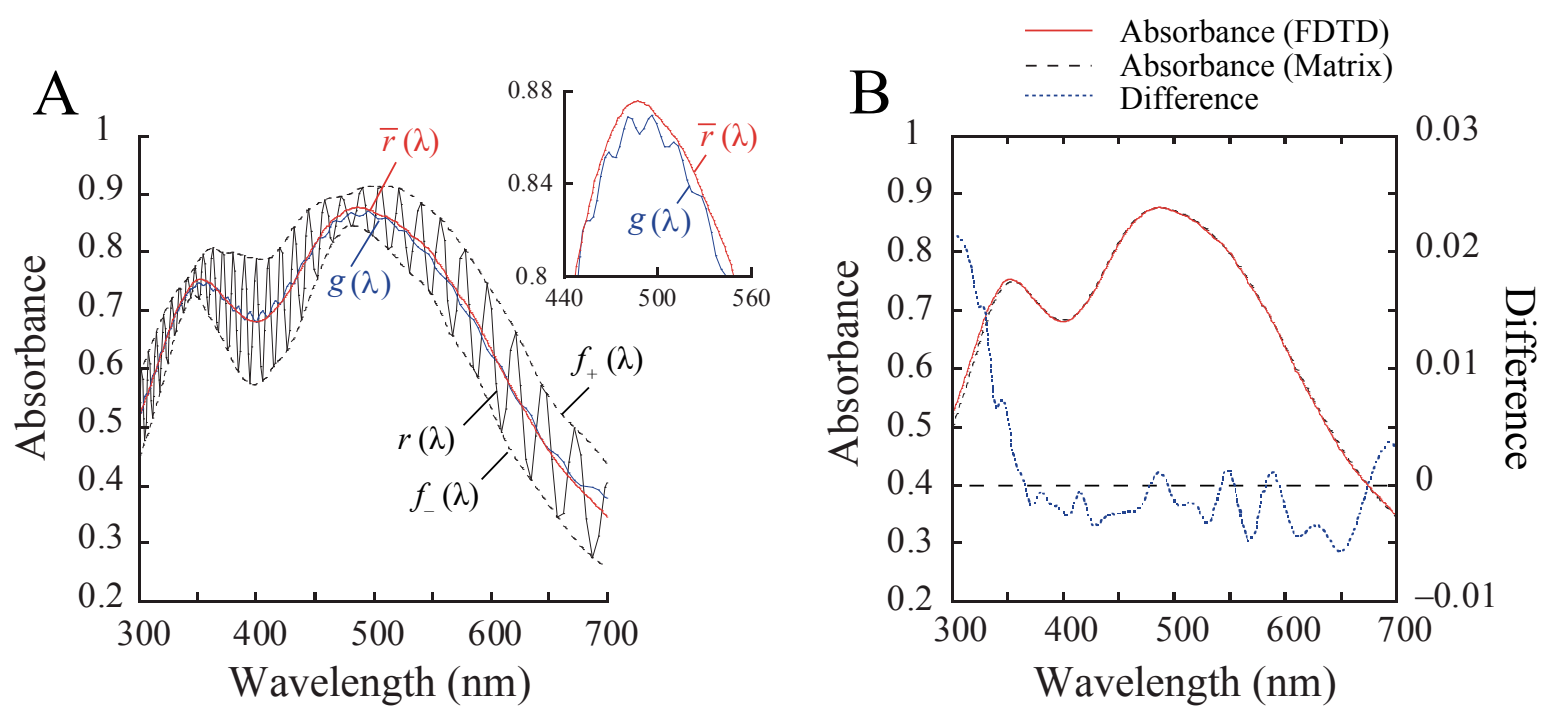

Fig. 2. (A) An example of the artificial interference effect in the absorbance in the active layer of OPVs. The functions of $r(\lambda)$ (black solid), $g(\lambda)$ (blue), $f_{+}(\lambda)$ and $f_{-}(\lambda)$ (black dashed), and $\bar{r}(\lambda)$ are plotted (the inset shows the magnification of $g(\lambda)$ and $\bar{r}(\lambda)$ ). (B) The absorbance spectrum obtained by the FDTD with the envelope method (red) and the characteristic matrix-based method (black). Blue line: the difference between the red and black lines. 
(1) Let us define $r_{i}$ for $i=1, \ldots, N$ to be the value of response $r(\lambda)$ at wavelength $\lambda_{i}$. We take the average of the response over nearby $p$ points at each wavelength $\lambda_{i}$, i.e., $\tilde{r}_{i}=$ $\frac{1}{p} \sum_{j=i-(p-1) / 2}^{i+(p-1) / 2} r_{j}$, and define $g(\lambda)$ (Fig. 2A, blue line) as the continuous function determined by the linear interpolation of the $N$ points $\left(\lambda_{i}, \tilde{r}_{i}\right)$ for $i$ $=1, \ldots, N$. (The parameter $p=21$, unless otherwise stated.)

(2) For all $i$, let $d_{i}$ be the minimum distance between the point $P_{i}\left(\lambda_{i}, r_{i}\right)$ and the curve of $g(\lambda)$ in the $\lambda-r$ plane. In addition, let $l_{i}$ be a value determined by $l_{i}=d_{i}$ for $r_{i}>\tilde{r}_{i}$ and $l_{i}=-d_{i}$ for $r_{i}<\tilde{r}_{i}$. Further, let $\Gamma_{+}$and $\Gamma_{-}$ be a set of integers $i$ for which $l_{i}$ takes a local maximum and minimum, respectively, i.e., $\Gamma_{+}=$ $\left\{i \mid l_{i}>l_{i-1}\right.$ and $\left.l_{i}>l_{i+1}\right\}$ and $\Gamma_{-}=\left\{i \mid l_{i}<\right.$ $l_{i-1}$ and $\left.l_{i}<l_{i+1}\right\}$.

(3) Let us define $f_{+}(\lambda)$ and $f_{-}(\lambda)$ as the functions generated by the cubic spline interpolation of the points $P_{i}\left(\lambda_{i}, r_{i}\right)$ for $i \in \Gamma_{+}$ and $i \in \Gamma_{-}$, respectively. The curves of $f_{+}(\lambda)$ and $f_{-}(\lambda)$ become the upper and lower envelopes of $r(\lambda)$, respectively, as shown in the dashed lines in Fig. 2A. The average of the two curves, represented by $\bar{r}(\lambda)=\left(f_{+}(\lambda)+f_{-}(\lambda)\right) / 2$, gives the response curve in which the artificial interference effect has been eliminated (Fig. 2A, red line).

For comparison to the envelope method, we attempted to remove the artificial interference effect from the FDTD response by two other methods as follows. The first method is the simple averaging, in which the linear interpolation of the points $\left(\lambda_{i}, \tilde{r}_{i}\right)$ with $\tilde{r}_{i}$

$=\frac{1}{p} \sum_{j=i-(p-1) / 2}^{i+(p-1) / 2} r_{j}$ (for $\left.i=1, \ldots, N\right)$ in (1) (shown above) gives the prediction of the response curve in the absence of artificial interference. The second method is the usage of low-pass filtering (LPF). In the LPF method, the frequency components higher than a threshold $f_{C}$ (in $\mathrm{nm}^{-1}$ ) is removed from $r(\lambda)$, and the lower frequency components give the predicted response curve, in which the artificial interference has been removed.

\section{Results and Discussion}

We first performed FDTD analysis for the OPV device without ARS. In the case that light passes through a glass substrate, strong oscillation emerges in the FDTD response due to the artificial interference effect, as mentioned above [11,12]. In order to remove the artificial interference from the spectrum of absorbance in the active layer, we applied the envelope method (see Experimental) (Fig. 2A).

In Fig. 2B, the absorbance spectrum obtained by using FDTD with the envelope method (Fig. 2B, red solid line) was compared with the spectrum obtained by the characteristic matrix-based method (Fig. 2B, black dashed line). The figure shows that the absorbance spectra obtained by the different methods agree very well for all wavelengths. As shown in Fig. 2 (blue dotted line), the difference between them is less than about 0.005 , except for a range of quite short wavelengths $(\lambda<350 \mathrm{~nm})$.

To quantify the difference between the absorbance in the active layer estimated by the FDTD and characteristic matrix-based methods, we define a measure $D$ as follows:

$$
D=\left(\frac{1}{\lambda_{1}-\lambda_{0}} \int_{\lambda_{0}}^{\lambda_{1}}\left[A_{F D T D}(\lambda)-A_{\text {matrix }}(\lambda)\right]^{2} d \lambda\right)^{1 / 2}
$$

Here, $\left[\lambda_{0}, \lambda_{1}\right]\left(\lambda_{0}=300 \mathrm{~nm}, \lambda_{1}=700 \mathrm{~nm}\right)$ is the wavelength range of interest. $A_{F D T D}(\lambda)$ and $A_{\text {matrix }}(\lambda)$ represent the absorbance spectra obtained by the FDTD and characteristic matrix-based analysis, respectively.

The $D$ value was calculated by applying the envelope method to the absorbance spectrum in the active layer of the OPVs in both the presence and absence of moth eye coating (Fig. 3A). Here, the period of moth eye array was set to be $32 \mathrm{~nm}$, because the application of EMT to the textured pattern requires that its period is small compared to the wavelength, as mentioned above. The height $H$ of moth eye was assumed to be 150 or $300 \mathrm{~nm}$. We used various values of $p$, which is an adjustable parameter in the envelope method (see Experimental), and plotted the $D$ values as function of $p$. Note that there are no other major parameters to be adjusted in the envelope method. Similarly, we applied the other possible algorithms to remove the artificial interference (see Experimental) and the $D$ values were calculated with changing the major parameters used in these algorithms. In Fig. 3B, the simple averaging method was used with various parameter values of $p$, and the relationship between $D$ and $p$ was plotted. In Fig. 3C, the LPF method was employed with various values of the threshold frequency $f_{C}$, and the $D$ values were plotted as function of 
$f_{C}$. The comparison of Figs. 3A-3C suggests that the variation in $D$ caused by changing the related parameters is much smaller when the envelope method is used than when the other methods are used. Further, the minimum values of $D$ obtained by changing these parameters are the smallest when the envelope method is applied for both the cases with and without moth eye (Table 1). These results suggest that the envelope method is not only accurate in estimating the optical response without artificial interference but also robust against changes in the related parameters, as compared to the other methods.

Furthermore, we applied the envelope method to the OPV with moth eye texture whose height and refractive index are changed (Fig. 4). As shown in Fig. 4A, the measure $D$ was at a low level $(\sim 0.006)$ for all the examined conditions, and did not change significantly. We also compared the level of $J_{S C}$ obtained by the envelope method and that obtained by the characteristic matrix-based method (Fig. 4B). In addition, we calculated the relative difference in $J_{S C}$ estimated by the different methods, which is defined $\delta_{J s c}=\left[\left(J_{S C}\right.\right.$ obtained by FDTD with the envelope method $) /\left(J_{S C}\right.$ obtained by the characteristic matrix-based method) - 1] $\times 100(\%)$ (Fig. 4C). The figure shows that, for all the cases, the value of $\left|\delta_{J s c}\right|$ is smaller than $0.5 \%$, which is much smaller than the alteration in $J_{S C}$ produced by the moth eye coating ( $\sim 9 \%$ in ref. [12]). This result suggests that the FDTD simulation integrated with the envelope algorithm is accurate enough to be used for the optical design and analysis of the moth eye antireflection for OPVs.

\section{Conclusion}

The FDTD simulation is widely used for analyzing the optical properties of textured surfaces such as moth eye structure. However, when FDTD is applied to OPVs, the existence of a thick glass substrate produces strong oscillatory response because of the artificial interference effect [12]. In this study, we investigated the envelope method, a computational algorithm to remove theartificial interference from the FDTD response. The results obtained by using the envelope method was compared with the other two algorithms, i.e., the simple averaging and LPF. It was revealed that the envelope method is the most accurate for estimating the optical
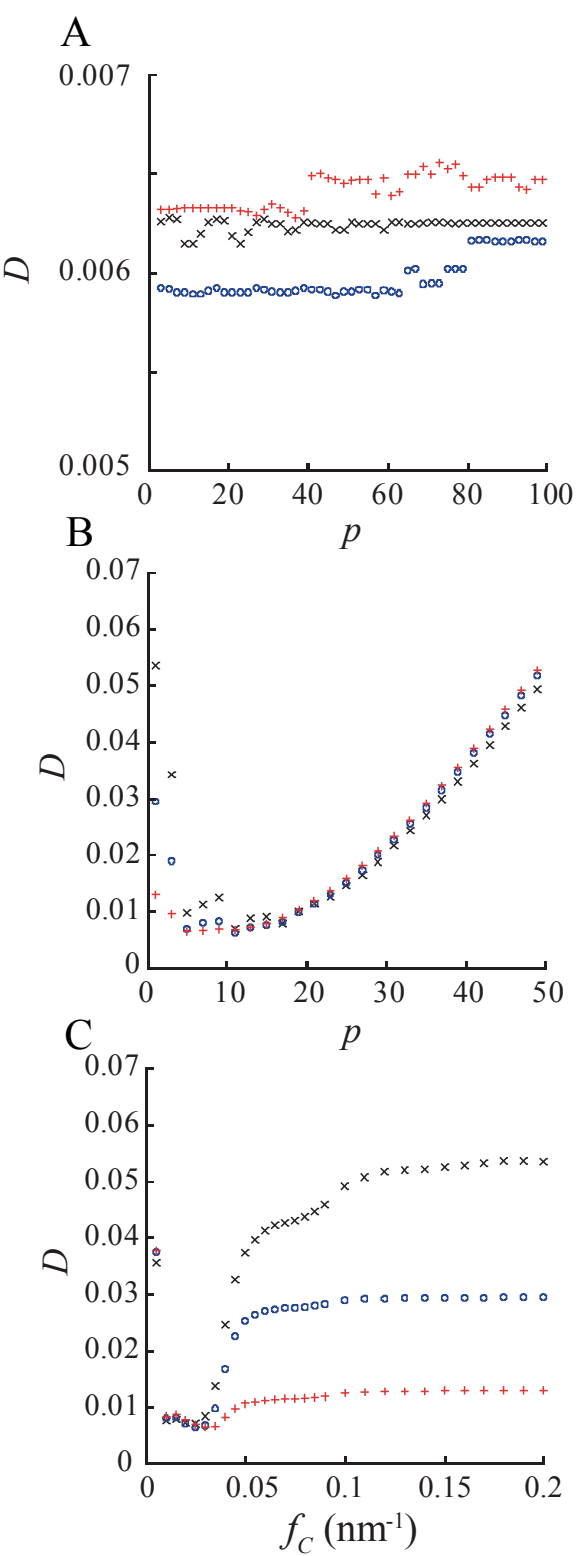

Fig. 3 The changes in the measure $D$ associated with the changes in control parameters, when the methods of the envelope (A), simple averaging (B), or LPF (C) are applied to the spectrum of absorbance in the active layer of OPVs. The black symbols show the case without ARS. The colored symbols represent the cases with moth eye coating for the height $H=150 \mathrm{~nm}$ (blue) and $300 \mathrm{~nm}$ (red).

response of OPVs and the most robust against variations in the related parameters. The FDTD simulation with the envelope method will be applicable to design antireflection surfaces for various solar cell devices, including OPVs, in which a light passes through a glass substrate.

\section{Acknowledgements}

This study was partially supported by 
Table 1. Minimum values of $D$ obtained by the envelope, simple averaging, and LPF methods for both the cases with and without moth eye.

\begin{tabular}{cccc} 
& Envelope & $\begin{array}{c}\text { Simple } \\
\text { averaging }\end{array}$ & LPF \\
\hline Without moth eye & $6.14 \times 10^{-3}$ & $6.86 \times 10^{-3}$ & $7.07 \times 10^{-3}$ \\
\hline With moth eye $(H=150 \mathrm{~nm})$ & $5.88 \times 10^{-3}$ & $6.31 \times 10^{-3}$ & $6.37 \times 10^{-3}$ \\
\hline With moth eye $(H=300 \mathrm{~nm})$ & $6.28 \times 10^{-3}$ & $6.54 \times 10^{-3}$ & $6.52 \times 10^{-3}$ \\
\hline
\end{tabular}

KAKENHI (26390025) from the Japanese government, and by JST, CREST.

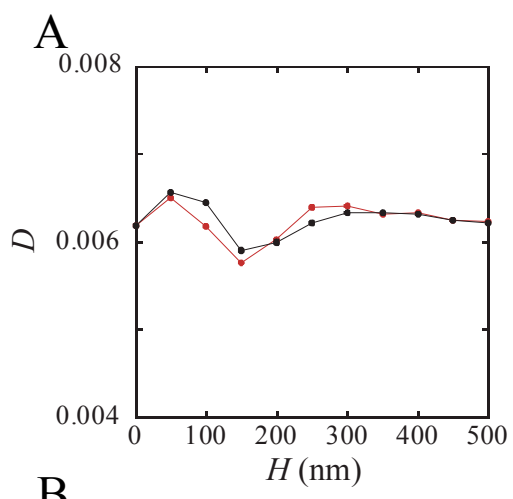

B

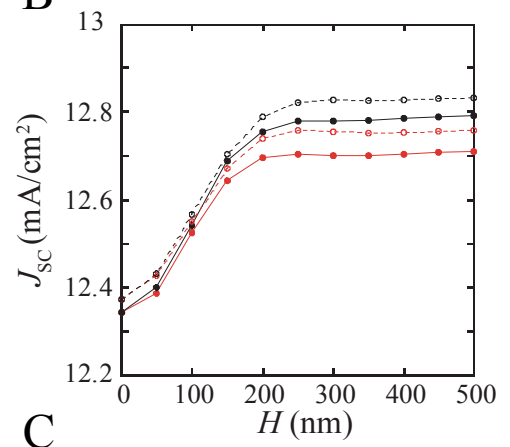

C

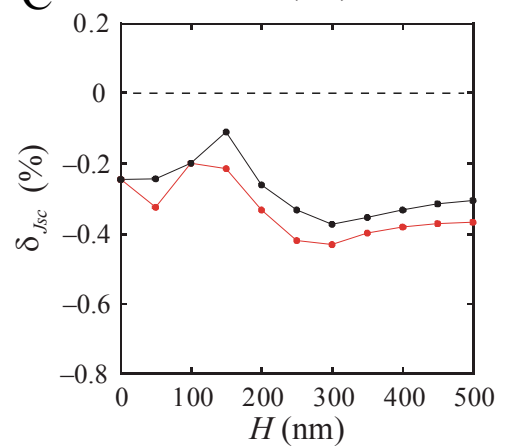

Fig. 4. (A) The $D$ value as function of the height $H$ of moth eye coating, when the envelope method is applied to the absorbance spectrum of the active layer. (B and C) The changes in $J_{\mathrm{SC}}$ obtained by the FDTD with the envelop method (B, solid lines) and the characteristic matrix-based method $(\mathrm{B}$, dashed lines), and the relative difference $\delta_{\text {Jsc }}$ between them (C) are plotted as function of $H$. In (A)-(C), the black and red lines show the cases where the refractive index of moth eye array is 1.5 (black) and 1.3 (red), respectively.

\section{References}

1. G. Yu, J. Gao, J. C. Hemmelen, F. Wudl and
A. J. Heeger, Science, 270 (1995) 1789.

2. S. H. Park, A. Roy, S. Beaupre, S. Cho, N. Coates, J. S. Moon, D. Morses, M. Leclerc, K. Lee and A. J. Heeger, Nat. Photonics, 3 (2009) 297.

3. M. Jorgensen, K. Norrmann, S. A. Cevorgyan, T. Tromholt, B. Andreasen and F. C. Krebs, Adv. Mater., 24 (2012) 580 .

4. K. Forberich, G. Dennler, M. C. Scharber, K. Hingerl, T. Fromherz and C. J. Brabec, Thin Solid Films, 516 (2008) 7167.

5. S. A. Boden and D. M. Bagnall, Prog. Photovolt. Res. Appl., 18 (2010) 195.

6. R. Brunner, O. Sandfuchs, C. Pacholski, C. Morhard and J. Spatz, Laser Photonics Rev., 6 (2012) 641.

7. A. Deinega, I. Valuev, B. Potapkin and Y. Lozovik, J. Opt. Soc., Am. A, 28 (2011) 770.

8. F. Jiao, Q. Huang, W. Ren, W. Zhou, F. Qi, Y. Zheng and J. Xie, Microelectron. Eng., 103 (2013) 126.

9. A. Taflove and S. C. Hagness, "Computational Electrodynamics: The Finite-Difference Time-Domain Method", Artech House Inc., Norwood, 2005.

10. L. Yang, Q. Feng, B. Ng, X. Luo and M. Hong, Appl. Phys. Exp., 3 (2010) 102602.

11. J. Wang, C. W. Wang, Y. Li, F. Zhou and W. M. Liu, Physica B, 405 (2010) 2061.

12. S. Kubota, K. Kanomata, B. Ahmmad, J. Mizuno and F. Hirose, J. Coat. Technol. Res., 13 (2016) 201.

13. H. A. Macleod, "Thin-Film Optical Filters", 4th ed. CRC Press, Boca Raton, 2010 .

14. A. D. Rakic, A. B. Djurisic, J. M. Elazar, and M. L. Majewski, Appl. Opt., 37 (1998) 5271.

15. D. E. Aspnes, Thin Solid Films, 89 (1982) 249.

16. A. Y. Darkwi, W. K. Lote and K. Ibrahim, Sol. Energy Mater. Sol. Cells., 60 (2000) 1. 